\title{
Self-bound models of compact stars and recent mass-radius measurements
}

\author{
M. G. B. de Avellar and J. E. Horvath" \\ Instituto de Astronomia, Geofísica e Ciências Atmosféricas \\ Rua do Matão 1226, 05508-900 São Paulo SP, Brazil \\ L. Paulucc 团 \\ Universidade Federal do $A B C$ \\ Rua Santa Adélia, 166, \\ 09210-170 Santo André, SP, Brazil
}

(Dated: July 12, 2018)

\begin{abstract}
The exact composition of a specific class of compact stars, historically referred to as "neutron stars", is still quite unknown. Possibilities ranging from hadronic to quark degrees of freedom, including self-bound versions of the latter have been proposed. We specifically address the suitability of strange star models (including pairing interactions) in this work, in the light of new measurements available for four compact stars. The analysis shows that these data might be explained by such an exotic equation of state, actually selecting a small window in parameter space, but still new precise measurements and also further theoretical developments are needed to settle the subject.

PACS numbers: 97.60.Jd, 26.60.Kp, 21.65.Qr
\end{abstract}

\section{INTRODUCTION}

The first reasonable ideas about the composition of compact stars stated that matter under extreme densities is composed of hadrons, i.e., mainly neutrons with small fractions of protons and also electrons. Further theoretical developments and modern experimental results opened the window to other possibilities as the existence of hyperons inside such stars due to the high densities, and a whole new class of condensates (see, for example, [1] and references therein). Another possibility arisen in the '70s is the existence of deconfined quarks inside compact objects, either located only in the inner core of these stars or present up to their surfaces. The latter extreme possibility was suggested about three decades ago [2 6], emerging as an astrophysical realization of the stability scenario of the so-called strange matter.

Consequences of the so-called strange matter hypothesis for compact stars have been extensively analyzed within the simple framework of the MIT Bag Model [7, 8] and the Nambu-Jona-Lasinio model [9 11] (see [12] for an overview on the subject). After an initial round of perturbative considerations [13, 14], the possibility of nonperturbative pairing between quarks [9, 15-19] allowed a new view on this subject due to a great enhancement on the window of stability for strange quark matter (having the strange quark mass, bag constant and the novel pairing energy of the quark condensate as parameters in this approach). This state of totally paired 3-flavor quarks is called the color-flavor locked (CFL) strange quark matter, in which quarks form Cooper pairs, thus lowering further the energy of the system.

\footnotetext{
*Electronic address: foton@astro.iag.usp.br

${ }^{\dagger}$ Electronic address: laura.paulucci@ufabc.edu.br
}

Much work has been put forward in order to have a better understanding on the actual composition of neutron stars, both observational and theoretical. Nevertheless, there is no absolute conclusion for any model as yet.

We intend in this work to contribute to the understanding of how the new and much more precise astrophysical measurements of the mass and radius of neutron stars 20 23] can help revealing the viability of exotic quark star models. We analyze in particular the viability of the class of CFL strange quark matter models. They are likely to be the most favorable candidates for self-bound stars, in spite that more complex models can be devised (for example intermediate phases such as LOFF may be present [24 26]), and they would constitute a benchmark in testing exotic compact star composition.

In the rest of this work we quantitatively show that accurate astrophysical measurements can already constrain important parameters of QCD, like the Cooper pair energy gap $(\Delta)$ and the strange quark mass $\left(m_{s}\right)$, and show how this is done given a subset of the present data.

\section{ANALYSIS}

When considering unpaired strange quark matter ( $u d s$ matter) the equation of state (EoS) assumes the simplest form when $m_{s} \rightarrow 0: \epsilon=3 P+4 B$, being $\epsilon, P$ and $B$, the energy density, pressure and bag constant, respectively. The non-zero strange quark mass has the effect of increasing the energy of the system [7]. On the other hand, if the system presents pairing interactions between quarks it has the net effect of lowering the energy of the system, making it more stable.

There have been different approaches to analyze this effect. Here, in order to set a framework for the class of models studied, we adopt the MIT bag model thermodynamic potential of CFL strange quark matter [16, 18] in 
bulk to order $\Delta^{2}$, being $\Delta$ the pairing energy gap for the phase, given by

$$
\Omega_{C F L}=\Omega_{f r e e}-\frac{3}{\pi^{2}} \Delta^{2} \mu^{2}+B
$$

In this expression, the CFL state is taken as a model composed of quark matter without pairing plus a term corresponding to the energy of the diquark condensate. Being so, the pairing energy gap is taken as a free parameter, instead of being defined in a self-consistent manner (see, for example, 27] for further discussions and more consistent models). The thermodynamic potential for the unpaired state is then

$\Omega_{\text {free }}=\sum_{i=u, d, s} \frac{1}{4 \pi^{2}}\left[\mu_{i} \nu\left(\mu_{i}^{2}-\frac{5}{2} m_{i}^{2}\right)+\frac{3}{2} m_{i}^{4} \log \left(\frac{\mu_{i}+\nu}{m_{i}}\right)\right]$

with $\mu=\left(\mu_{u}+\mu_{d}+\mu_{s}\right) / 3$ and the common Fermi momentum, $\nu=2 \mu-\sqrt{\mu^{2}+m_{s}^{2} / 2}$.

The EoS is finally obtained by relating the energy density $\epsilon$ to the pressure $P$ as follows

$$
\epsilon=3 \mu n_{B}-P
$$

with the particle density given by $n_{B}=\left(\nu^{3}+2 \Delta^{2} \mu\right) / \pi^{2}$, and the pressure $P=-\Omega_{C F L}$.

Due to the fact that $m_{s} \neq 0$, the EoS have to be computed numerically. However, the CFL EoS shows a remarkably linear behavior [19]. Because of this, much work has been done in order to construct a simple EoS that includes the effects of pairing and a heavy strange quark, keeping the linear behavior to a certain extent. We start our analysis, then, by postulating a linear EoS for CFL matter.

We have parameterized the equation of state for CFL matter as

$$
P=\alpha\left(c^{2} \rho-4 B_{e f f}\right)
$$

where $\alpha$ is a parameter related with the stiffness of the EoS and $B_{\text {eff }}$ is certainly related to the bag constant $B$ and gap parameter $\Delta$. Within this approximation, it is easy to show that the Tolman-Oppenheimer-Volkoff (TOV) and the EoS equations can be written in dimensionless forms:

$$
\begin{gathered}
p_{\star}^{\prime}=-\frac{m_{\star} \rho_{\star}}{x^{2}}\left(1+\frac{p_{\star}}{\rho_{\star}}\right)\left(1+\frac{4 \pi x^{3} p_{\star}}{m_{\star}}\right)\left(1-2 \frac{m_{\star}}{x}\right)^{-1} \\
m_{\star}^{\prime}=4 \pi x^{2} \rho_{\star}
\end{gathered}
$$

$$
p_{\star}=\alpha\left(\rho_{\star}-1\right)
$$

where the starred quantities are the dimensionless mass, pressure and mass density; $x$ is the dimensionless radius and $\alpha \equiv \frac{1}{3+\beta}$ is an effective parameter which depends on the quantities $\Delta$ and $m_{s}$ in a complicated but very mild and continuous way.

Our main goal here was to devise an easy way to display the location (locus) of the maximum mass and its correspondent radius, e. g., fitting a curve $f=f(x, \alpha)$. This locus strongly depends, in our parameterization, on $\alpha$. The higher its value, the higher the maximum mass. Because of this, we needed to construct several mass-radius relationships varying $\alpha$ within an acceptable range, in this case, from 0.20 to 0.36 . To comply with observations, we needed to reach at least $2 M_{\odot}$. We then fitted an expression for the maximum mass $\left(m_{\star, \max }\right)$ of each sequence and their correspondent radius $\left(x_{c o r r}\right)$ dependent on $\alpha$, yielding the result

$$
\begin{gathered}
m_{\star, \max }=-0.163 \alpha^{2}+0.201 \alpha+0.00286 \\
x_{\text {corr }}=-0.262 \alpha^{2}+0.329 \alpha+0.111 .
\end{gathered}
$$

Restoring the units, we finally find the relation

$$
M_{\max }=R_{\text {corr }} \frac{c^{2}}{G} \frac{m_{\max }}{x_{\text {corr }}} .
$$

given that

$$
r=x \sqrt{\frac{c^{4}}{4 B_{e f f} G}} \quad m=m_{\star} \sqrt{\frac{c^{8}}{4 B_{e f f} G^{3}}} .
$$

Here is the point where the dependence on $B_{\text {eff }}$ appears. Notice that the radius and the mass individually depend on $B_{\text {eff }}$ but the relation between maximum mass and its correspondent radius (when the physical units are restored) does not: it depends only on the value of $\alpha$. This feature is already present in Witten's original paper and seems to be generic for any linear equation of state.

From the effective parameters found above, we have analyzed the agreement of the mass-radius relation obtained for different sets of parameters with data for the following stars: 4U 1608-522 [20], EXO 1745-248 [21], 4U 1820-30 222] and PSR J1614-2230 [23]. The results are presented in the following section.

\section{RESULTS}

The value of $\alpha$ as a function of $B_{\text {eff }}$ can be constrained by using the observed mass of PSR J1614-2230, considering that the maximum mass provided by a specific EoS 
should at least equal the mass of this heavy star. This can be obtained from eq. (8)-(10) or by varying the values of $\alpha$ and $B_{\text {eff }}$ on Eq. (44) and constructing the corresponding mass-radius sequences. Within this previously determined region and having generated the mass-radius relations for the effective equation of state using these constrained values, we compared them with the other three stars within a $3 \sigma$ demanded precision. The effective parameters, i.e. all the pairs $\left(\alpha, B_{e f f}\right)$, that would be suitable for explaining all four data at the same time, i.e. the observed masses and radii and their respective error bars, are those corresponding to the line shown on the left panel of Fig. 1] The initial and final points of this line were used to generate the mass-radius relations shown on the right panel of the same Fig.

We finally find a very narrow window for $\alpha$ and $B_{\text {eff }}$ that can provide compatibility with all four measurements together. As a consequence, the mass-radius relations shown on the right panel of Fig. 1 are almost completely overlapping.

We would like now to relate the effective parameters determined through this analysis with the free parameters determining the CFL matter state. Unfortunately, although the EoS presents a linear behavior, the linearization of Eq.(11) is not possible analytically, unless some major approximations are made. Without the latter, one cannot directly relate $\alpha$ and $B_{e f f}$ to $\Delta, m_{s}$ and $B$. Previous attempts to find such a relation include the work of Alford et al. [28], where a form of parameterized EoS was proposed, in which QCD corrections to the pressure, represented by a parameter $c \approx 0.3$, have been added. This parameterization relied on a power series expansion in $\mu$ for the thermodynamic potential of the quark phase, reading

$$
\Omega_{Q M}=-\frac{3}{4 \pi^{2}} a_{4} \mu^{4}+\frac{3}{4 \pi^{2}} a_{2} \mu^{2}+B_{e f f}
$$

with $a_{4}=1-c$ and $a_{2}$ as free parameters. In their approach, the strange quark mass and gap parameter affect $a_{2}$ alone and $B_{\text {eff }}$ is determined by the density at which the transition between quark and nuclear matter phases occur, as they were interested in addressing hybrid stars. Although they have been able to write $a_{2}=a_{2}\left(m_{s}, \Delta\right)$, and one can in fact for CFL quark matter in equilibrium (within this framework) separate the thermodynamic potential expression to order $m_{s}^{4}$ with only three terms proportional to $\mu^{4}, \mu^{2}$ and $\mu^{0}$, the chemical potential itself has a nonlinear and nontrivial dependence on all other parameters, as shown in details in [19]. We have chosen not to make this approximation here, so that we are not able to write $\alpha$ and $B_{\text {eff }}$ as function of $m_{s}, B$ and $\Delta$ in analytical closed form.

Since it is not possible to obtain the expression relating the parameterized EoS to the CFL one, in order to verify the effectiveness of our own linearization, we have taken the full equation of state for CFL strange quark matter, as written in Eq. (11)-(3), and analyzed the agreement of the mass-radius relation obtained for the same set of stars as before. We have covered acceptable ranges for the free parameters: the bag constant $B>57 \mathrm{MeV} / \mathrm{fm}^{3}$, the superconductor gap $0 \leq \Delta \lesssim 50 \mathrm{MeV}$ and the strange quark mass $m_{s} \geq 100 \mathrm{MeV}$. Recent measurements give a very precise value for the $s$ quark mass at the energy scale of $2 \mathrm{GeV}: 92.4 \pm 1.5 \mathrm{MeV}$ [29]. At a smaller energy scale this value should be even higher (see [30], Particle Properties - quark masses, and references therein) so we decided to adopt the value of $100 \mathrm{MeV}$ as a lower boundary.

After solving the TOV equations for several sets of $\left(m_{s}, B, \Delta\right)$ and then comparing the values of mass and radius with the measurements under consideration, the best agreement found is the one giving the mass-radius relation shown in Fig. 2, The "optimal" values of the parameters found are $B \sim 69 \mathrm{MeV} / \mathrm{fm}^{3}, \Delta \sim 50 \mathrm{MeV}$, and $m_{s} \sim 120 \mathrm{MeV}$ (well within the window of stability for CFL strange quark matter, thus justifying the quark star scenario). Notice, however, that for $4 \mathrm{U} 1820-30$ the mass-radius curve is in agreement within $\sim 3.4 \sigma$ only. It should also be noticed, nevertheless, that one cannot state that in this case the $3 \sigma$ boundary is the one corresponding to $99.9 \%$ of compatibility, since there are very few degrees of freedom involved in the analysis. Therefore, the agreement can be considered as acceptable.

We see that, although the equation of state for CFL strange quark matter clearly presents a linear behavior in the pressure versus energy density dependency, when a parameterization of the type $P=\alpha\left(c^{2} \rho-4 B_{e f f}\right)$ is made, the coefficients $\alpha$ and $B_{\text {eff }}$ cannot be treated as independent from each other. Since we are working with a "toy model", in which one considers a system without any pairing plus a term corresponding to the condensate energy, $\Delta$ surely adds up to the bag constant but has no influence on the "unpaired state". However, the role of the strange quark mass is more subtle. In the analysis discussed in [28], the authors show that $B_{\text {eff }}$, besides being related to the vacuum pressure (and, in the CFL phase, also to the gap parameter), incorporates an additional term which is proportional to $m_{s}^{4}$ when the power series expansion is made. So even in a simplified scheme, we see that $B_{\text {eff }}$ is not dependent on the bag constant alone but should also incorporate additional terms which are important for determining the stiffness of the EoS.

In this way, even having found values for the parameters in the linearization that could fit the data within a $\sim 3 \sigma$ region, when a full equation of state is employed, one cannot reach such a good agreement. This kind of problem is also evident in the oldest expression employed by Benvenuto and Horvath [31] $P=\frac{1}{3+a}\left[c^{2} \rho-(4+b) B\right]$, the complicated dependence of the parameters on $m_{s}$, $B$ and $\Delta$ (and hence their correlation) affects the accuracy of the $M_{\max }-R$ locus and blurs to some extent an accurate determination of the parameter space.

Another general aspect worth of noticing is the very soft raise of the mass-radius curve for paired strange quark matter. It could be attributed to the simplic- 

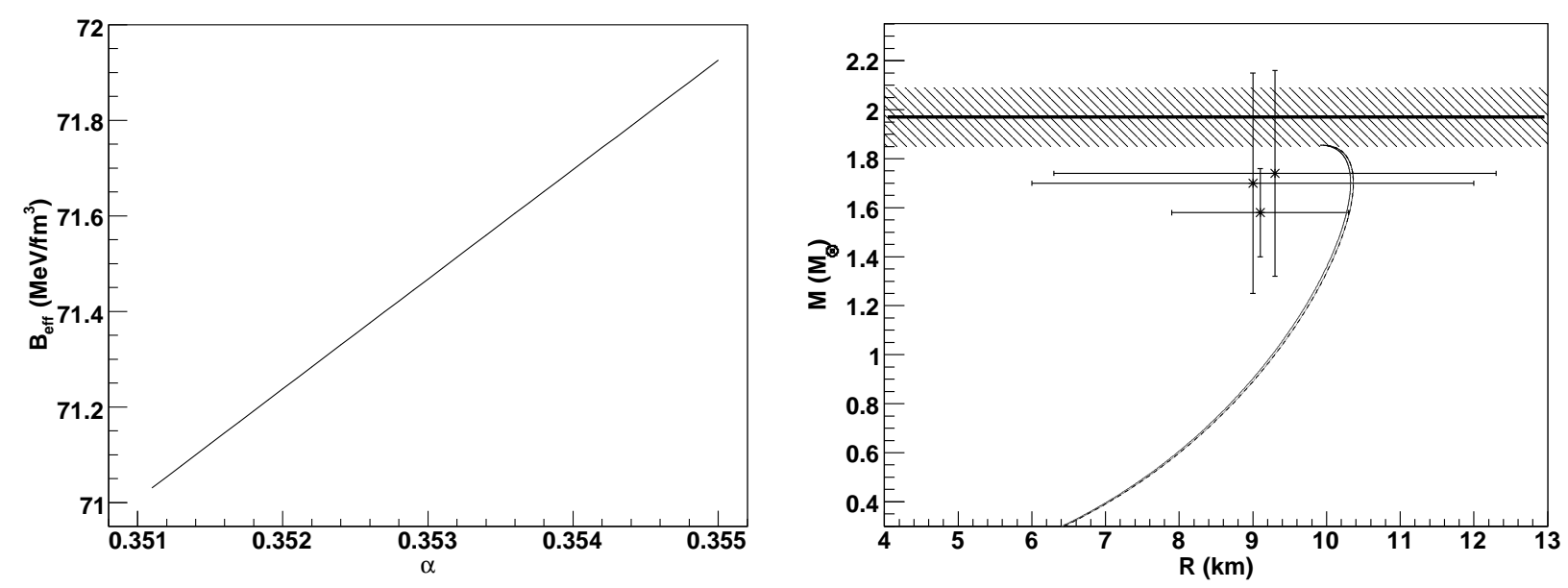

FIG. 1: On the left panel, effective parameters allowing compatibility within $3 \sigma$ with recent data (taking into account the observed masses and radii and their respective error bars). On the right, mass-radius measurements are shown as crosses with $3 \sigma$ error bars. Data from PSR J1614-2230 is shown as a shaded region, since there is only information on the mass for this object. The two mass-radius relations shown correspond to the lower and upper limits of $\alpha$ and $B_{\text {eff }}$ that can explain the data (shown on the right).

ity of the model, not considering interactions between quarks other than pairing and one-gluon exchange (see 7] for a discussion on the scaling of the bag parameter with $\alpha_{c} \neq 0$ ). A steeper raise of the mass-radius relation could indeed provide a better agreement with the observed data and perhaps an increase in the maximum allowed mass. This could be an important issue since it would be impossible to reach masses as high as $2 M_{\odot}$, for example, with a single quark matter EoS that would also need to explain the values for measured masses and radii for the stars $4 \mathrm{U} 1608-522$, EXO 1745-248, and $4 \mathrm{U}$ 1820-30, if they happen ultimately to be accurate and correct.

There have been claims, however, that the values of masses and radii for $4 \mathrm{U} 1608-522$, EXO $1745-248$ and $4 \mathrm{U}$ 1820-30 have been mistakenly derived 32]. The computational method used in the previous analysis was criticized pointing out possible systematic errors which substantially reduce the uncertainties in the masses and radii values for these systems. Considering in turn the determinations given by Steiner, Lattimer and Brown [32], we have repeated the previous calculations to verify whether the CFL equation of state is compatible with the reconstruction of the data presented by them. The results of our analysis for this case are very different, though. We see that the very narrow parameter space previously found now becomes an extremely wide region of possibilities, as depicted in Fig 3 .

The error bars for these systems, according to Steiner, Lattimer and Brown, are much bigger than previously considered and all three stars are clustered with close values for their masses and radii $\left(M \sim 1.5 M_{\odot}\right.$ and $R \sim 11$ $\mathrm{km}$ with more visible differences for the mass when the photospheric radius for photon emission is considered to

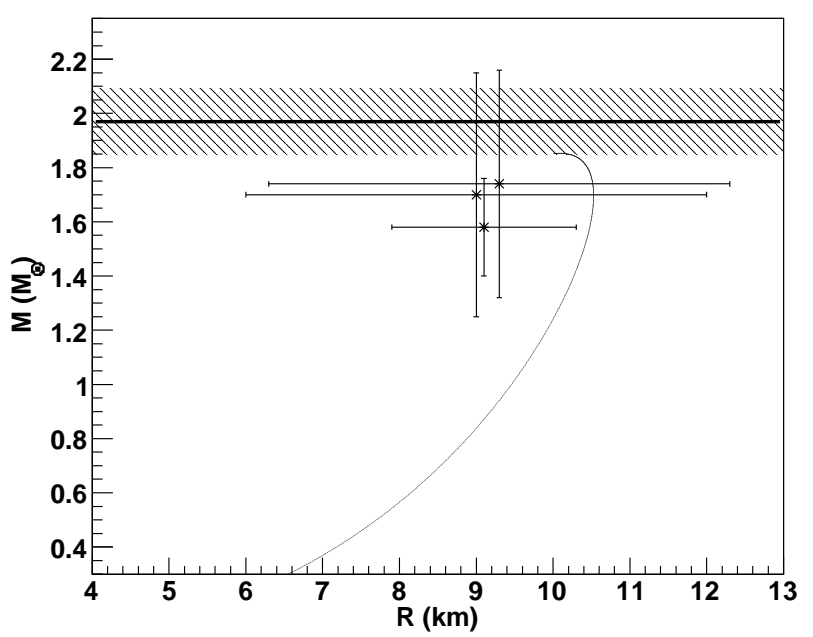

FIG. 2: Best agreement for the mass-radius relation with recent measurements (data represented as in Fig. 1) considering the CFL equation of state. Values for the parameters are $B \sim 69 \mathrm{MeV} / \mathrm{fm}^{3}, \Delta \sim 50 \mathrm{MeV}$, and $m_{s} \sim 120 \mathrm{MeV}$.

be much larger than the stellar radius, $R_{p h}>>R$, the color correction $f_{c}$ is a key ingredient for this behavior). This leads now to a large set of parameters $m_{s}, B$ and $\Delta$ that could in principle explain the data, basically having the mass of PSR J1614-2230 as the main quantity providing the constrain for the equation of state. In other words, one can find several sets of (B, $\left.\Delta, m_{s}\right)$ that satisfy the necessary conditions within the values needed in this analysis. The general behavior is to have a lower maximum mass with an increase in the strange quark mass 

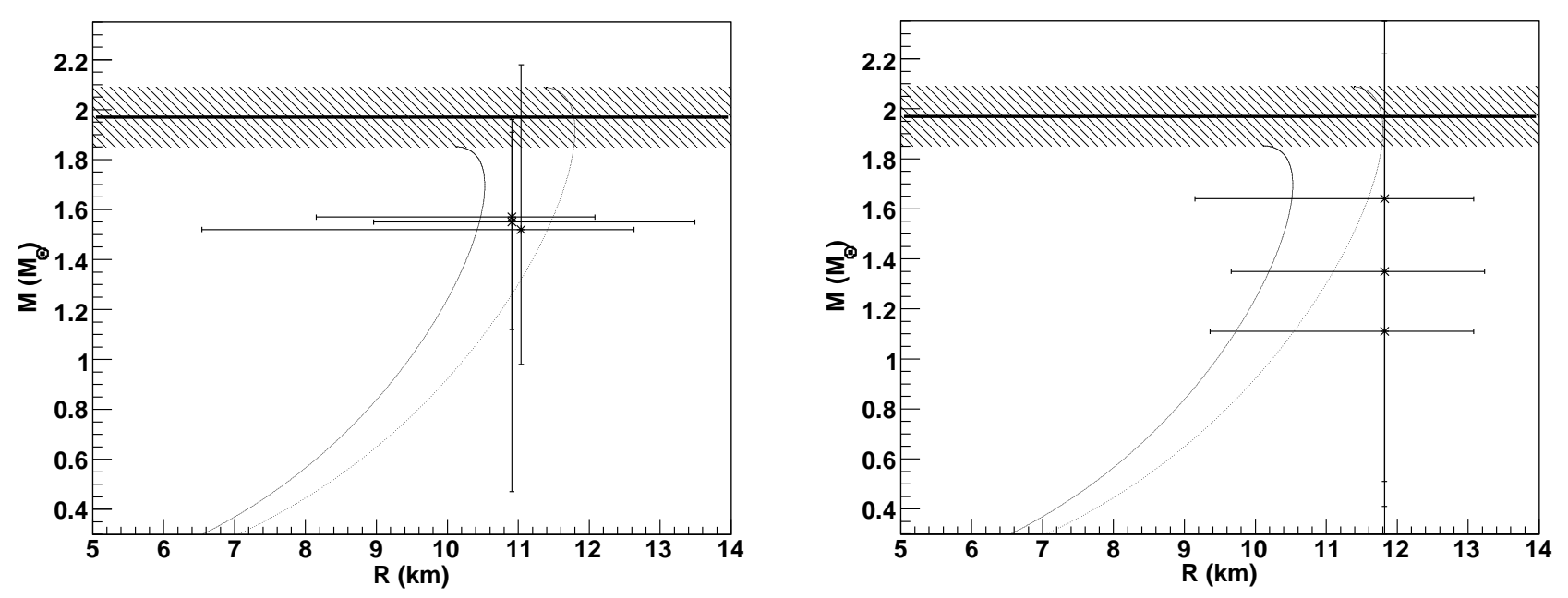

FIG. 3: A second evaluation of the reconstructed data as given in 32. On the left side, mass and radius of $4 \mathrm{U} 1608-522$, EXO 1745-248 and 4U 1820-30 and mass range for PSR J1614-2230 considering $R=R_{p h}$. On the right, the same for $R>>R_{p h}$ (see text for further details). Two examples of mass-radius relations for CFL stars are shown for the sets of parameters $\left(B, \Delta, m_{s}\right)$ given by $\left(69 \mathrm{MeV} / \mathrm{fm}^{3}, 50 \mathrm{MeV}, 120 \mathrm{MeV}\right)$ and $\left(58 \mathrm{MeV} / \mathrm{fm}^{3}, 62 \mathrm{MeV}, 110 \mathrm{MeV}\right)$ to highlight the fact that, in this case, the mass-radius relation is constrained solely by the massive pulsar.

and bag constant when the values of the other two variables are held fixed. As the gap parameter increases, the the maximum allowed mass also increases.

\section{CONCLUSIONS}

Precise measurements for masses and radii of compact stars are very important for constraining their composition. However, besides being precise, it is necessary that they also be accurate for a clear comparison of the massradius relation coming from very different compositions with data.

Given the present controversy of the radius determination quoted in the paper, this appears to be the main issue to be resolved for an advance of the conclusions of studies like ours. Meantime we can only conclude that only with small error bars for both mass and radii determinations (as in the analysis of [20 22]) the parameters of the CFL equation of state can be effectively constrained. Otherwise, several sets of parameters defining the CFL EoS can be used to match the data. In this case, more advanced detection techniques and physical analysis are required to constrain the radius $R_{p h}$ in X-ray burst models.

Since one should not treat $\alpha$ and $B_{\text {eff }}$ as independent quantities in a parameterized EoS for CFL strange quark matter like the one proposed here, an analysis performed with a parameterization with correlated coefficients should be explored. We intend to address this subject in a future work.

An additional interesting report is the very low mass of at least one neutron star mass observed ( $M=0.87 \pm$ $0.07 M_{\odot}$ for $\left.4 \mathrm{U} 1538-52\right)$ [33], actually not well explained by ordinary stellar evolution arguments. This "low-end" mass extreme is also important for the quest of exotic compositions in their interiors. In fact, confirming such a measurement would imply a radius of $R \leq 9 \mathrm{~km}$ within the self-bound hypothesis, while the measurement of, say, $R \geq 11 \mathrm{~km}$ would be totally inconsistent with it, pointing to a normal composition. We are at the edge of finally addressing the fundamental composition of compact stars, at least on a first level that leaves a handful of possibilities left out of a huge number of proposals.

\section{Acknowledgments}

We wish to acknowledge the support of the Fundação de Amparo à Pesquisa do Estado de São Paulo and the CNPq Agency and CAPES Agency (Brazil). We would also like to thank Dr. Franciole Marinho for very useful discussions on statistical analysis.
[1] F. Weber, Prog. Part. Nucl. Phys. 54, 193 (2005).

[2] N. Itoh, Progr. Theor. Phys. 44, 291 (1970).

[3] A. Bodmer, Phys. Rev. D 4, 1601 (1971).
[4] S. A. Chin and A. K. Kerman, Phys. Rev. Lett. 43, 1292 (1979).

[5] H. Terazawa, Tokyo U. Report. pp. INS-336 (1979). 
[6] E. Witten, Phys. Rev. D 30, 272 (1984).

[7] E. Farhi and R. L. Jaffe, Phys. Rev. D 30, 2379 (1984).

[8] C. Alcock, E. Farhi, and A. V. Olinto, Astrophys. Journal 310, 261 (1986).

[9] M. Alford, K. Rajagopal, and F. Wilczek, Nuc. Phys. B537, 433 (1999).

[10] O. G. Benvenuto and G. Lugones, Int. J. Mod. Phys. 7, 29 (1998).

[11] S. Yin and R.-K. Su, Phys. Rev. C 77, 055204 (2008).

[12] M. Buballa, Phys. Rept. 407, 205 (2005).

[13] D. Bailin and A. Love, Phys. Rep. 107, 325 (1984), and references therein.

[14] J. E. Horvath, O. G. Benvenuto, and H. Vucetich, Phys. Rev. D 44, 3797 (1991).

[15] R. Rapp, T. Schaefer, E. V. Shuryak, and M. Velkovsky, Ann. Phys. (NY) 280, 35 (2000).

[16] M. Alford, K. Rajagopal, S. Reddy, and F. Wilczek, Phys. Rev. D 64, 074017 (2001).

[17] K. Rajagopal and F. Wilczeck, arXiv:hep-ph/0011333, and references therein.

[18] K. Rajagopal and F. Wilczek, Phys. Rev. Lett. 86, 3492 (2001).

[19] G. Lugones and J. E. Horvath, Phys. Rev. D 66, 074017 (2002).

[20] T. Guver, F. Ozel, A. Cabrera-Lavres, and P. Wroblewski, Astrophys. J. 712, 964 (2010), arXiv:0811.3979v4 [astro-ph].

[21] F. Ozel, T. Guver, and D. Psaltis, Astrophys. J. 693, 1775 (2009).

[22] T. Guver, P. Wroblewski, L. Camarota, and F. Ozel, Astrophys. J. 719, 1807 (2010).

[23] P. B. Demorest, T. Pennucci, S. M. Ransom, M. Roberts, and J. W. T. Hessels, Nature 467, 1081 (2010).

[24] M. Alford, J. A. Bowers, and K. Rajagopal, Phys. Rev. D 63, 074016 (2001).

[25] J. A. Bowers and K. Rajagopal, Phys. Rev. D 66, 065002 (2002).

[26] R. Casalbuoni and G. Nardulli, Rev. Mod. Phys. 76, 263 (2004).

[27] L. Paulucci, E. J. Ferrer, V. de la Incera, and J. E. Horvath, Phys. Rev.D 83, 043009 (2011).

[28] M. Alford, M. Braby, M. Paris, and S. Reddy, Astrophys. J. 629, 968 (2005).

[29] C. T. H. Davies et al., Phys. Rev. Lett 104, 132003 (2010).

[30] K. Nakamura et al., J. Phys. G 37, 075021 (2010).

[31] O. G. Benvenuto and J. E. Horvath, Mon. Not. R. astr. Soc. 241, 43 (1989).

[32] A. W. Steiner, J. M. Lattimer, and E. F. Brown, Astrophys. J. 722, 33 (2010).

[33] M. L. Rawls et al., Astrophys. J. 730, 25 (2011). 